\title{
Propriedades Físicas e de fluxo de cultivares de feijão para o dimensionamento de silos verticais
}

\author{
Physical and flow properties of common bean cultivars for the design of vertical silos \\ Propiedades físicas y de flujo de cultivares de frijol común para el diseño de silos verticales
}

Recebido: 08/09/2021 | Revisado: 14/09/2021 | Aceito: 15/09/2021 | Publicado: 18/09/2021

\author{
Diego José Araújo Bandeira \\ ORCID: https://orcid.org/0000-0002-6245-9875 \\ Universidade Federal de Campina Grande, Brasil \\ E-mail: diegoimperium8@gmail.com \\ Marcia Janiele Nunes da Cunha Lima \\ ORCID: https://orcid.org/0000-0003-3697-8724 \\ Universidade Federal de Campina Grande, Brasil \\ E-mail: marciacunhalima87@hotmail.com \\ Antônio Nunes de Oliveira \\ ORCID: https://orcid.org/0000-0001-5697-8110 \\ Universidade Federal de Campina Grande, Brasil \\ E-mail: nunes.vieira@ifce.edu.br \\ Rodolfo Ramos Castelo Branco \\ ORCID: https://orcid.org/0000-0002-9034-7985 \\ Universidade Federal de Campina Grande, Brasil \\ E-mail: castelobrancorodolfo@gmail.com \\ Kaline Ligia do Nascimento \\ ORCID: https://orcid.org/0000-0002-9831-7166 \\ Universidade Federal de Campina Grande, Brasil \\ E-mail: kaline.dmi@hotmail.com \\ Raniere Fernandes Costa \\ ORCID: https://orcid.org/0000-0002-5831-4251 \\ Universidade Federal de Campina Grande, Brasil \\ E-mail: ranierengenharia.costa @gmail.com \\ José Jefferson da Silva Nascimento \\ ORCID: https://orcid.org/0000-0002-2620-6491 \\ Universidade Federal de Campina Grande, Brasil \\ E-mail: jeffpesquisador@gmail.com
}

\begin{abstract}
Resumo
A produção agrícola brasileira excede a capacidade armazenadora, com isso a construção de silo se torna imprescindível. s características físicas e de fluxo do produto analisado é essencial no projeto de silo. Diante disto o presente trabalho tem como objetivo determinar as propriedades físicas e de fluxo de diferentes cultivares de feijão para dimensionamento de silos verticais. O experimento foi conduzido no Laboratório de Construções Rurais e Ambiência- LACRA da Universidade Federal de Campina Grande, no município de Campina Grande-PB, analisando os diferentes tipos de feijões: carioquinha (Phaseolus vulgaris L.), preto (Phaseolus vulgaris L.) e macassar (Vigna unguiculata). Foram aferidas as características físicas (teor de umidade, granulometria, densidade) e de fluxo (ângulo e efetivo ângulo de atrito interno, coesão média e ângulo de atrito existente entre o produto e diferentes materiais parede do silo), foram utilizados os seguintes materiais de parede: acrílico, aço liso, aço rugoso, alumínio, madeira, analisando a melhor opção. Concluiu-se que os produtos são classificados como grãos grossos, com fluxo livre, para inclinação da tremonha houve diferença numérica entre as paredes analisadas.
\end{abstract}

Palavras-chave: Phaseolus vulgaris L; Silos verticais; Vigna unguiculata.

\begin{abstract}
The Brazilian agricultural production exceeds the storage capacity, with that the construction of a silo becomes essential. he physical and flow characteristics of the analyzed product is essential in silo design. Therefore, this work aims to determine the physical and flow properties of different common bean cultivars for dimensioning vertical silos. The experiment was conducted at the Laboratory of Rural Constructions and Ambience - LACRA of the Federal University of Campina Grande, in the city of Campina Grande-PB, analyzing the different types of beans: carioquinha (Phaseolus vulgaris L.), black (Phaseolus vulgaris L.) and macassar (Vigna unguiculata). The physical characteristics (moisture content, granulometry, density) and flow (angle and effective internal friction angle, average cohesion and friction angle existing between the product and different silo wall materials) were measured. wall: acrylic, smooth
\end{abstract}


steel, rough steel, aluminum, wood, analyzing the best option. It was concluded that the products are classified as coarse grains, with free flow, for hopper inclination there was a numerical difference between the analyzed walls.

Keywords: Phaseolus vulgaris L; Vertical silos; Vigna unguiculata.

\begin{abstract}
Resumen
La producción agrícola brasileña supera la capacidad de almacenamiento, por lo que la construcción de un silo se vuelve imprescindible. Las características físicas y de flujo del producto analizado son fundamentales en el diseño del silo. Por lo tanto, este trabajo tiene como objetivo determinar las propiedades físicas y de flujo de diferentes cultivares de frijol común para dimensionar silos verticales. El experimento se realizó en el Laboratorio de Ambientes y Construcciones Rurales - LACRA de la Universidad Federal de Campina Grande, en la ciudad de Campina GrandePB, analizando los diferentes tipos de frijol: carioquinha (Phaseolus vulgaris L.), negro (Phaseolus vulgaris L.) y macassar (Vigna unguiculata). Se midieron las características físicas (contenido de humedad, granulometría, densidad) y flujo (ángulo y ángulo de fricción interna efectiva, cohesión promedio y ángulo de fricción existente entre el producto y los diferentes materiales de la pared del silo). Pared: acrílico, acero liso, acero rugoso, aluminio, madera, analizando la mejor opción. Se concluyó que los productos se clasifican como granos gruesos, con flujo libre, para la inclinación de la tolva hubo diferencia numérica entre las paredes analizadas.
\end{abstract}

Palabras clave: Phaseolus vulgaris L; Silos verticales; Vigna unguiculata.

\title{
1. Introdução
}

A produção de grãos cresce anualmente de forma acentuada devido ao aumento da produtividade das culturas, a produção de grãos no Brasil de acordo com a Companhia Nacional de Abastecimento, estima-se para safra 2020/21 um total de 268,7 milhões de toneladas, um acréscimo de 11 milhões de toneladas em relação à safra 2019/20 (CONAB, 2021). O Brasil possui uma limitada capacidade de armazenamento, que não acompanha a produção agrícola do país, o que proporciona perdas econômicas para os produtores e, desta forma, a construção de silos verticais surge como uma alternativa para solução deste problema (Bandeira et. al., 2020).

O feijão (Phaseolus vulgaris L.) é uma importante fonte de proteínas, fibras, ferro, carboidratos, minerais e vitaminas para milhões de pessoas, tanto nos países em desenvolvimento como nos países desenvolvidos (Lin et al., 2008).

Estima-se que existam 55 espécies do gênero Phaseolus (Carneiro, 2005). Esse gênero compreende todas as espécies conhecidas como feijão, sendo a Phaseolus vulgaris L. a mais conhecida e a que possui inúmeras variedades tais como, Carioca, Roxo, Mulatinho, Preto, entre outras (Pires et al., 2005).

Os silos verticais são estruturas comumente utilizadas pelas indústrias, setor agrícola e mineral para armazenar e conservar a granel, produtos sólidos granulares ou pulverulento (Dornelas et. al., 2021). Os silos verticais (graneleiros) são amplamente utilizados no Brasil e possibilitam recursos de movimentação de carga e descarga mecanizada, como elevadores e correias transportadoras, máquinas de limpeza e secadores, sistemas de aeração e termometria (Baroni et al., 2017).

Os silos verticais apresentam-se como soluções alternativas, entretanto, grande número dos silos existentes não apresenta condições ideais de operação devido ao insuficiente conhecimento das pressões que variam no tempo e no espaço interno, do fluxo e das variáveis que afetam o comportamento dos produtos armazenados. Tal situação tem contribuído para os acidentes e colapsos em silos (Dornelas et. al., 2021).

O Fluxo de massa é mais vantajoso visto que elimina a possibilidade de obstrução do fluxo, é uniforme e fácil de controlar, a densidade durante a descarga é praticamente constante e toda a capacidade de armazenamento é aproveitada, uma das poucas vantagens do fluxo de funil é o menor desgaste nas paredes do silo, uma vez que o atrito entre a parede e o produto durante o descarregamento é desprezível, as pressões as quais as paredes são submetidas são menores, reduzindo a redução do custo de construção (Amóros et al., 2002; Oginni \& Fasina, 2018; Olivares et al., 2018; Fullard et al., 2020).

A principal dificuldade enfrentada pelas indústrias que trabalham com grãos e pulverulentos destaca-se a formação de arcos coesivos que impedem, parcial ou completamente, a descarga, que está intrinsecamente relacionada às geometrias dos 
silos e tremonhas bem como as propriedades físicas e de fluxo dos produtos sólidos e condições ambientais como: pressão atmosférica, temperatura e umidade relativa (Lopes Neto \& Nascimento, 2013).

Um dos problemas mais significativos em projeto de silos é a exata predição da distribuição de cargas no corpo do silo, com especial atenção para as pressões exercidas nas paredes em virtude do empuxo do produto armazenado. Essas distribuições de pressão dependem do comportamento do produto, da interação entre o produto armazenado e da parede do silo e, ainda, das propriedades de fluxo durante o processo de carregamento e descarregamento (Martinez et al., 2002; Costa, 2013; Tascón, 2017; Ayres et al., 2020).

\section{Metodologia}

\section{Localização do Experimento}

O experimento será conduzido no Laboratório de Construções Rurais e Ambiência- LACRA da Universidade Federal de Campina Grande, no município de Campina Grande-PB, localizado no interior do estado da Paraíba.

\section{Produto Analisado}

Foram determinadas as propriedades físicas e de fluxo dos feijões carioquinha (Phaseolus vulgaris L.), preto (Phaseolus vulgaris L.) e macassar (Vigna unguiculata) (Figura 1).

Figura 1. Tipos de feijão analisados, (a) Feijão carioquinha, (b) Feijão preto e (c) Feijão macassar.

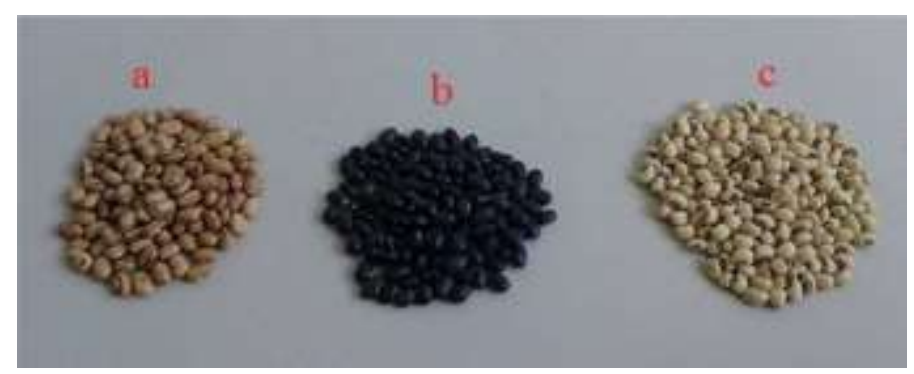

Fonte: Acervo do Autor.

\section{Teor de Umidade}

O teor de umidade foi determinado através do método padrão de estufa, com circulação de ar à $105 \pm 3{ }^{\circ} \mathrm{C}$, durante 24 horas, conforme descrito em regras para Análise de Sementes (BRASIL, 2009).

\section{Granulometria}

A granulometria foi determinada por um agitador mecânico de peneiras (Figura 2), utilizando 500 gramas de produto com uma frequência do aparelho de $10 \mathrm{~Hz}$, durante 5 minutos e peneiras de padrão $\mathrm{ABNT}(9,52 ; 4,76 ; 4,00 ; 2,8 \mathrm{~mm})$ em ordem decrescente de malha, pesando ao final o material que permanecerá em cada peneira determinando, assim, sua percentagem. 
Figura 2. Agitador mecânico de peneiras.

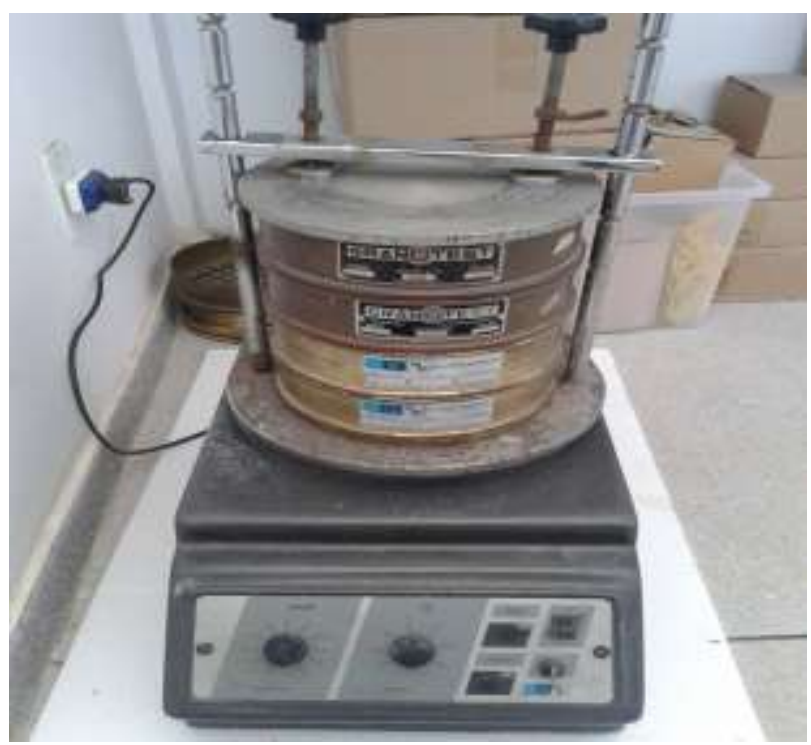

Fonte: Acervo do Autor.

\section{Caracterização do produto pelas propriedades de fluxo}

Foram determinadas as propriedades de fluxo: peso específico consolidado $(\gamma)$, ângulo de atrito interno $(\phi)$, efetivo ângulo de atrito interno $(\delta)$ e ângulo de atrito do produto com a parede $\left(\phi_{\mathrm{w}}\right)$. Adotando a metodologia recomendada pela OPERATING INTRODUCTION FOR THE TRANSLATIONAL SHEAR TESTER (TSG - 70/140) utilizando o aparelho “Jenike Shear Cell” (Figura 3).

Figura 3. Jenike utilizado para determinação das propriedades de fluxo.

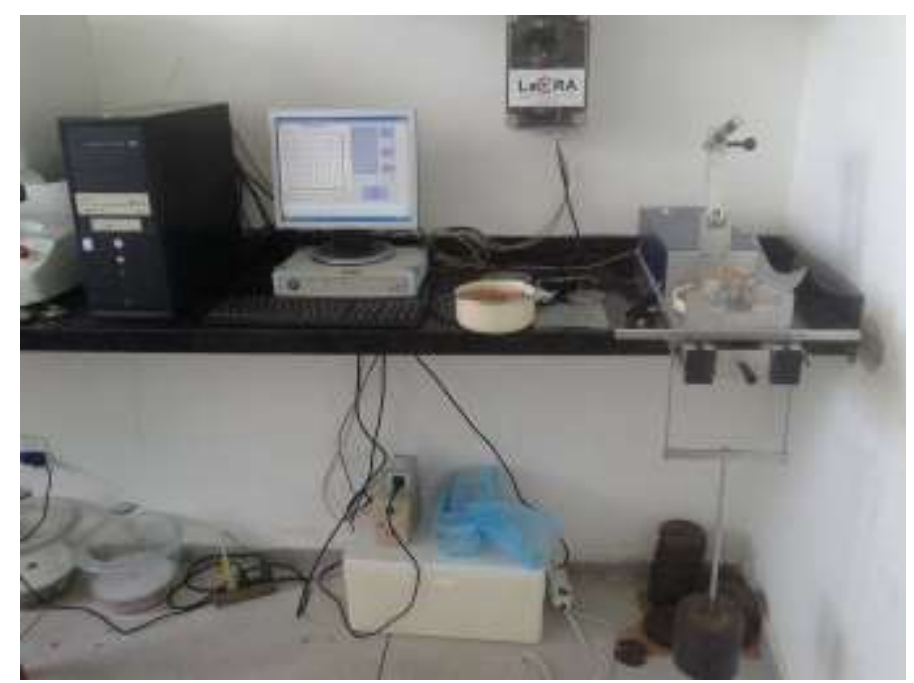

Fonte: Acervo do Autor.

Foram feitos dois ensaios: cisalhamento do produto e cisalhamento do produto com o material da parede (madeira, aço liso, aço rugoso, acrílico e alumínio), a fim de determinar o ângulo de atrito interno, efetivo ângulo de atrito interno, o ângulo de atrito com a parede e a coesão do produto.

Para realização do ensaio de cisalhamento todas as cargas utilizadas no experimento estão descritas na Tabela 1. 
Tabela 1. Níveis de carga utilizados nos ensaios de cisalhamento.

\begin{tabular}{c|ccc}
\hline Pré-cisalhamento $(\mathrm{Kg})$ & \multicolumn{3}{c}{ Cisalhamento $(\mathrm{Kg})$} \\
\hline 10 & 7 & 5 & 3,5 \\
7 & 5 & 3,5 & 2 \\
5 & 3,5 & 2 & 1 \\
\hline
\end{tabular}

Fonte: Acervo do Autor.

Utilizando um programa de computação gráfica (Auto CAD) com os resultados, foram traçados os gráficos de tensão normal e versus tensão de cisalhamento, para as cargas utilizadas, traçando círculos de Mohr.

As equações utilizadas para obtenção dos pontos que originaram a reta de coesão, para construção dos círculos de Mohr onde foram mensuradas as tensões normais de consolidação e inconfinada, estão representadas abaixo:

$$
\begin{array}{cc}
\gamma=\frac{W_{t}-\left(W_{b}+W_{r}+W_{L}\right)}{V_{t}} & \tau_{s}=\tau \frac{\bar{\tau}_{p}}{\tau_{p}} \\
\tau_{p}=\frac{S_{p}}{A} g & \tau=\frac{S_{s}}{A} g \\
\sigma_{p}=\left(\frac{H+W_{p}+W_{r}+W_{L}+\gamma V_{r}}{A}\right) g & \bar{\tau}=\frac{\sum_{1}^{n} \tau_{p}}{n} \\
\sigma_{s}=\left(\frac{H+W_{s}+W_{r}+W_{L}+\rho_{b} V_{r}}{A}\right) g & \overline{\sigma_{p}}=\frac{1}{n}
\end{array}
$$

Sendo,

$\mathrm{W}_{\mathrm{t}} \quad$ Peso total da célula com o produto

$\mathrm{W}_{\mathrm{b}} \quad$ Peso da base do anel

$\mathrm{W}_{\mathrm{r}} \quad$ Peso do anel de cisalhamento

$\mathrm{W}_{\mathrm{L}} \quad$ Peso da tampa

$\mathrm{V}_{\mathrm{t}} \quad$ Volume total da célula de cisalhamento

$\mathrm{V}_{\mathrm{r}} \quad$ Volume correspondente ao anel de cisalhamento

A Área da seção transversal do anel de cisalhamento

G Aceleração da gravidade, $9,81 \mathrm{~m} / \mathrm{s}^{2}$ 
$\mathrm{S}_{\mathrm{s}} \quad$ Força de cisalhamento/inicio de consolidação

$\mathrm{W}_{\mathrm{p}} \quad$ Peso no pendural durante o pré-cisalhamento

$\mathrm{W}_{\mathrm{S}} \quad$ Peso no pendural durante o cisalhamento

Para a determinação do ângulo de atrito com a parede foram utilizadas as seguintes equações:

$$
\sigma_{w}=\left(\frac{W_{w t}+W_{w}-W_{m}+H}{A}\right) g \quad \tau_{w}=\frac{S_{w}}{A} g \quad \varphi_{w}=\arctan \frac{\tau_{w}}{\sigma_{w}}
$$

Sendo,

$\emptyset_{\mathrm{w}} \quad$ Ângulo de atrito com a parede

$\mathrm{W}_{\mathrm{wt}} \quad$ Peso total da célula, inclusive anel, tampa e o produto

$\mathrm{W}_{\mathrm{m}} \quad$ Peso da chapa do material da parede

$\mathrm{W}_{\mathrm{w}} \quad$ Peso no pendural para o teste de atrito na parede

$\mathrm{S}_{\mathrm{w}} \quad$ Força de cisalhamento

\section{Resultados e Discussão}

Na Tabela 2, encontram-se os resultados dos ensaios granulométricos do feijão carioquinha, preto e macassar, com umidade de $2,91 \%, 3,53 \%$ e $3,03 \%$ (b.s.), respectivamente.

Tabela 2. Análise granulométrica das diferentes cultivares de feijão.

\begin{tabular}{llll}
\hline \multirow{2}{*}{ Cultivar } & Peneira & Retido & Acumulado \\
\cline { 2 - 4 } & $(\mathrm{mm})$ & $(\%)$ & $(\%)$ \\
\hline Carioquinha & 4,76 & 99,99 & 99,99 \\
& 4,00 & 0,01 & 100,00 \\
\hline \multirow{2}{*}{ Preto } & 4,76 & 99,79 & 99,79 \\
& 4,00 & 0,21 & 100,00 \\
\hline \multirow{2}{*}{ Macassar } & 4,76 & 99,86 & 99,86 \\
& 4,00 & 0,13 & 100,00 \\
\hline
\end{tabular}

Fonte: Autores.

Observou-se, que o maior percentual retido foi na peneira 4,76 mm $(99,99 \%, 99,79 \%, 99,86 \%)$ de material retido para o feijão carioquinha, preto e macassar, respectivamente. Com base nestes dados, de acordo com a classificação de produtos armazenáveis em função do diâmetro das partículas proposta pela norma AS 3774 (1996), classificam-se as cultivares como sendo um produto de grão grosso.

$\mathrm{Na}$ Tabela 3, estão apresentados os resultados das propriedades de fluxo das cultivares de feijão, obtidos a partir do círculo de Mohr confirmados nos gráficos demonstrados no Apêndice A. 
Tabela 3. Propriedades de fluxo dos produtos analisados.

\begin{tabular}{|c|c|c|c|c|c|c|c|}
\hline \multirow{2}{*}{ Cultivar } & \multicolumn{2}{|l|}{$\gamma\left(\mathrm{N} / \mathrm{m}^{3}\right)$} & \multicolumn{2}{|c|}{$\Phi \mathrm{e}\left(^{\circ}\right)$} & \multicolumn{2}{|c|}{$\Phi \mathrm{i}\left(^{\circ}\right)$} & \multirow{2}{*}{$\mathrm{C}(\mathrm{Pa})$} \\
\hline & inf & sup & $\inf$ & sup & inf & sup & \\
\hline Carioquinha & 7923,34 & 8072,12 & 28 & 30 & 29 & 31 & 250,667 \\
\hline Preto & 8055,04 & 8106,09 & 26 & 27 & 27 & 28 & 50 \\
\hline Macassar & 8008,54 & 8147,5 & 24 & 25 & 24 & 25 & 64 \\
\hline
\end{tabular}

$(\gamma)$ = Peso específico consolidado; $(\Phi \mathrm{i})$ = Ângulo de atrito interno; $(\Phi \mathrm{e})=$ Efetivo ângulo de atrito interno; $\mathrm{C}=\mathrm{Coesão}$; inf = limite inferior; $\sup =$ limite superior. Fonte: Autores.

Os resultados do peso específico consolidado $(\gamma)$ feijão carioquinha, preto e macassar apresentaram valores médios de $7991,3412 \mathrm{~N} / \mathrm{m}^{3}, 8086,1640 \mathrm{~N} / \mathrm{m}^{3}$ e $8058,8567 \mathrm{~N} / \mathrm{m}^{3}$, respectivamente. Relacionando os dados obtidos na pesquisa com o de normas internacionais, correlaciona-se o feijão com a soja por terem formato parecido e por serem leguminosas.

O peso específico consolidado médio do feijão carioquinha está dentro da norma AS 3774 (1996) e EUROCODE 1 (2006), onde o limite superior é de $8000 \mathrm{~N} / \mathrm{m}^{3}$, entretanto, o feijão preto e o macassar estão um pouco acima do limite estabelecido pelas normas.

Observou-se que os valores para o feijão carioquinha do ângulo de atrito interno inferior e superior, $29^{\circ}$ e $31^{\circ}$, respectivamente, e efetivo ângulo de atrito interno inferior $28^{\circ}$ e superior $30^{\circ}$, com valor de coesão média de $250,667 \mathrm{~Pa}$.

Com o feijão preto o ângulo de atrito interno inferior e superior, $27^{\circ}$ e $28^{\circ}$, respectivamente, e efetivo ângulo de atrito interno inferior $26^{\circ}$ e superior $27^{\circ}$, com valor de coesão média de $50 \mathrm{~Pa}$.

Para o feijão macassar o ângulo de atrito interno inferior e superior, $24^{\circ}$ e $25^{\circ}$, respectivamente, e efetivo ângulo de atrito interno inferior $24^{\circ}$ e superior $25^{\circ}$, com valor de coesão média de $64 \mathrm{~Pa}$.

Pode-se observar que o feijão preto e macassar apresentaram semelhança nos resultados, diferindo apenas do feijão carioquinha.

Correlacionando o feijão com a soja por terem formatos parecidos, os limites de efetivo ângulo de atrito interno estão dentro da norma AS 3774 (1996), que delimita o limite inferior em $25^{\circ}$ e o superior em $32^{\circ}$, para os feijões carioquinha e preto, sendo o efetivo ângulo de atrito interno encontrado para o feijão macassar $1^{\circ}$ inferior a norma, ou seja, $24^{\circ}$.

Ao correlacionar o feijão com a soja, nota-se que o ângulo de atrito interno para os feijões carioquinha e preto, estão acima da norma EUROCODE 1 (2006), onde determina o ângulo de atrito interno médio de $25^{\circ}$, entretanto foi observado que o feijão macassar está dentro da norma com um ângulo de atrito interno de $25^{\circ}$.

Para confecção da tremonha foram calculados seus ângulos de inclinação com a vertical apresentados na Tabela 4.

Tabela 4. Ângulos de inclinação da Tremonha com a horizontal.

\begin{tabular}{llll}
\hline & Inclinação da Tremonha $\left(^{\circ}\right)$ & \\
\hline Material & Feijão Carioquinha & Feijão Preto & Feijão Macassar \\
\hline Madeira & 25,84 & 23,08 & 19,42 \\
Acrílico & 26,32 & 22,88 & 19,29 \\
Aço Rugoso & 26,80 & 24,16 & 20,25 \\
Aço Liso & 26,25 & 25,37 & 20,42 \\
Alumínio & 26,48 & 22,34 & 22,01 \\
\hline
\end{tabular}


Para a construção da tremonha, quanto maior o ângulo de inclinação da tremonha com a horizontal mais fácil será o fluxo, com isso, observando os resultados de inclinação, para construção da tremonha para armazenamento de feijão carioquinha, o maior ângulo de inclinação foi utilizando o aço rugoso como material de composição, sendo o mesmo de $26,8^{\circ}$, por ser bem utilizado na construção de tremonhas indica-se seu uso.

Com a finalidade de armazenamento de feijão preto, o maior ângulo de inclinação da tremonha foi utilizando o aço liso como material de composição, sendo ele $25,37^{\circ}$, por ser um material normalmente utilizado na confecção de tremonhas ele é indicado.

Nota-se que para o armazenamento de feijão macassar, o maior ângulo de inclinação foi utilizando o alumínio como material de composição, sendo o mesmo de $22,01^{\circ}$, indica-se a utilização de alumínio na construção da tremonha.

\section{Conclusão}

Após análise dos resultados as cultivares de feijão analisadas foram classificadas em grãos grossos, como fluxo livre.

Todas as propriedades físicas e de fluxo para os matérias analisados foram estabelecidas e fomentam um acrescimento no banco de dados para futuras pesquisas.

Foi possível o dimensionamento do silo vertical, para todos os tipos de materiais de estudados, não apresentando nenhum problema dimensional.

Sugerimos para trabalhos futuros, a continuidade da produção de dados experimentais sobre propriedades físicas e de fluxo de diversos tipos materiais, para fomento de pesquisas futura, uma vez que estudos com simulação computacional se tornam cada vez mais comuns.

\section{Referências}

Ayres, G. D. J.,do Nascimento, J. W. B. \& Mascarenhas, N. M. H. (2020). Rompimento de arcos coesivos em silos verticais com emprego de pneumaticos: Uma revisão bibliográfica. Revista de Ciências Agrárias, 2020,43(4): 390-395.

Amorós, J. L. (2002). Projeto de Silos para o Armazenamento de Materiais na Forma de Pó. Problemas Associados à Etapa de Descarga. Revista Cerâmica Industrial, 7 (1), 12-23.

AS 3774. (1996). Australian Standard. Loads on bulk containers. Sydney.

Bandeira, D. J. A., Nascimento, J. J. S., \& Nascimento, J. W. B. (2020). Análise do fluxo de ração avícola em silos verticais esbeltos com insert de cone invertido. Research, Society and Development,9(11), e63091110369.

Baroni, G. D., Benedeti, P. H. \& Seidel, D. J. (2017). Cenários prospectivos da produção e armazenagem de grãos no Brasil. Revista Thema, 14(4), 55-64.

Brasil. (2009). Ministério da Agricultura, Pecuária e Abastecimento. Secretaria de Defesa Agropecuária. Regras para análise de sementes. Brasília: Mapa/ACS.

Carneiro, H. S. (2005). Comida e sociedade: significados sociais na história da alimentação. História: Questões \& Debates. 42(1), 71-80.

Conab -Companhia Nacional de Abastecimento (2021) Acompanhamento safra brasileira de grãos, v.8-Safra 2020/21, n. 4 -Quarto levantamento, Brasília, p. 1-85, janeiro 2021. ISSN 2318-7913. https://www.conab.gov.br/info-agro/safras/graos/boletim-da-safra-de-graos.

Costa, C. A. DA. (2013). Propriedades físicas, mecânicas e de fluxo de produtos agroindustriais. (Dissertação de Mestrado). Universidade Federal de Campina Grande, Campina Grande, PB.

Dornelas, K. C., Ayres, G. D. J., \& Nascimento, J. W. B. do. (2021). Emprego de inserts em silos metálicos: revisão sobre o padrão de fluxo dos produtos e distribuição das cargas na estrutura. Research, Society and Development, 10(4), e55710414580. https://doi.org/10.33448/rsd-v10i4.14580.

Dornelas, K. C., Ayres, G. D. J., Rodrigues, H. C. S., Nascimento, R. T. do., Lopes Neto, J. P., \& Nascimento, J. W. B. do. (2021). Gargalo da produção agrícola brasileira: Colapsos de silos verticais. Research, Society and Development, 10(8), e14510817253. https://doi.org/10.33448/rsd-v10i8.17253 .

Eurocode 1. (2006). Actions on structures - Part 4: Silos and tanks. Bruxelas.

Fullard, L.A.,Godfrey, A.J.R.,Manaf, M.F.,Davies, C.E.,Cliff, A. \& Fukuoka, M. (2020). Mixing experiments in 3D-printed silos,the role of wall friction and flow correcting inserts. Advanced Powder Technology, v. 31(5), 1915-1923. 
Research, Society and Development, v. 10, n. 12, e208101220347, 2021

(CC BY 4.0) | ISSN 2525-3409 | DOI: http://dx.doi.org/10.33448/rsd-v10i12.20347

Leite, P. G.; Lopes Neto, J. P.; Leite Filho, M. T.; Marques, J. I.; Martins, J. J. (2014). Características de fluxo do milho para o dimensionamento de silos verticais. CONTECC - Congresso Técnico Científico da Engenharia e da Agronomia.

Lin, L.; Harnly, J.; Pastor-Corrales, M. S.; Luthria, D. L. (2008). The polyphenolic profiles of common bean (Phaseolus vulgaris L.). Food Chemistry. 107(1), 399-410.

Lopes Neto, J. P.; Nascimento, J. W. B. (2013). Características de fluxo e projeto de tremonhas cônicas em silos verticais. Revista Brasileira de Engenharia Agrícola e Ambiental, 17(3), 339-345.

Martinez, M. A.; Alfaro, I.; Doblare, M. (2002). Simulation of axisymmetric discharging in metallic silos. Analysis of the induced pressure distribution and comparison with diferent standards. Engineering Structures, 24(12), 1561-1574.

Oginni, O. \& Fasina, O. (2018). Theoretical estimation of silo design parameters for fractionated loblolly pine grinds -Moisture content and particle size effects. Industrial Crops and Products, 123, 379-385.

Olivares, M. C. V.,Benito, J.G.,Uñac, R.O. \& Vidales, A. M. (2018). Towards a one parameter equation for a silo discharging model with inclined outlets. Powder Technology, 336, 265-272.

Pires, C. V.; Oliveira, M. G. A.; Cruz, G. A. D. R.; Mendes, F. Q.; De Rezende, S. T.; Moreira, M. A. (2005). Composição físico-química de diferentes cultivares de feijão (Phaseolus vulgaris L.). Alimentos e Nutrição, 16(2), 157-162.

Tascón, A. (2017) Design of silos for dust explosions: Determination of vent area sizes and explosion pressures. Engineering Structures, 134, 1-10. 